\title{
Exercise intensity and its impact on relationships between salivary immunoglobulin A, saliva flow rate and plasma cortisol concentration
}

\author{
Christof A. Leicht ${ }^{1} \cdot$ Victoria L. Goosey-Tolfrey ${ }^{1} \cdot$ Nicolette C. Bishop $^{1}$
}

Received: 16 June 2017 / Accepted: 14 March 2018 / Published online: 7 April 2018

(c) The Author(s) 2018

\begin{abstract}
Introduction Salivary secretory immunoglobulin A ( $\operatorname{sg}$ A), saliva flow rate and plasma cortisol concentrations have been shown to be influenced by exercise, particularly the intensity exercise is performed at, and circadian variation. The autonomic nervous system partly regulates salivary secretion, but it is not yet known whether cortisol also explains some variation in salivary parameters.

Methods Twelve moderately trained male individuals $\left(\dot{V} \mathrm{O}_{2 \text { peak legs }}: 46.2 \pm 6.8 \mathrm{~mL} \cdot \mathrm{kg}^{-1} \cdot \mathrm{min}^{-1}\right)$ performed three 45 -min constant load exercise trials in the morning: arm cranking exercise at $60 \% \dot{V} \mathrm{O}_{2 \text { peak arms }} ;$ moderate cycling at $60 \% \dot{V} \mathrm{O}_{2 \text { peak legs; }}$;

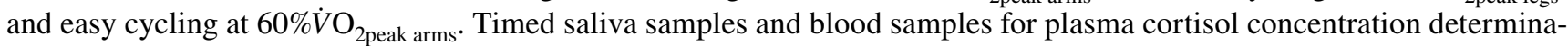
tion were obtained before, post, $2 \mathrm{~h}$ post, and $4 \mathrm{~h}$ post-exercise. Saliva was collected in an additional resting trial at the same time points.

Results At each time point for each exercise trial, negative correlations between cortisol and saliva flow rate (explaining $25 \pm 17 \%$ of the variance, $R^{2}=0.002-0.46$ ) and positive correlations between cortisol and sIgA concentration (explaining $8 \pm 8 \%$ of the variance $R^{2}=0.002-0.24$ ) were found. Saliva flow rate increased over time, whereas sIgA concentration and cortisol decreased over time for all trials $(P<0.05)$, there was no effect of time for sIgA secretion rate $(P=0.16)$.

Conclusion These results show a relationship between cortisol and saliva flow rate, which directly impacts on the concentration of salivary analytes. This study further confirms circadian variations in salivary parameters which must be acknowledged when standardising salivary data collection.
\end{abstract}

Keywords Salivary secretory immunoglobulin A $\cdot$ Mucosal immune function $\cdot$ Exercise intensity $\cdot$ Exercise modality . Upper body exercise

$\begin{array}{ll}\text { Abbreviations } \\ \text { ANOVA } & \text { Analysis of variance } \\ \text { ELISA } & \text { Enzyme-linked immunosorbent assay } \\ R & \text { Correlation coefficient } \\ R^{2} & \text { Coefficient of determination } \\ \mathrm{RPE} & \text { Rating of perceived exertion } \\ \mathrm{SD} & \text { Standard deviation } \\ \mathrm{SIgA} & \text { Salivary secretory immunoglobulin A } \\ \dot{V} \mathrm{O}_{2 \text { max }} & \text { Maximum oxygen uptake } \\ \dot{V} \mathrm{O}_{2 \text { peak }} & \text { Peak oxygen uptake }\end{array}$

Communicated by Fabio Fischetti.

Christof A. Leicht

c.a.leicht@lboro.ac.uk

1 The Peter Harrison Centre for Disability Sport, School of Sport, Exercise, and Health Sciences, Loughborough University, Loughborough LE11 3TU, UK

\section{Introduction}

Saliva flow rate and the secretion of various salivary compounds are under the control of the autonomic nervous system (Chicharro et al. 1998). Stimulation of sympathetic and parasympathetic neurons (Proctor and Carpenter 2007) or infusion of adrenergic agonists (Proctor et al. 2003) induce changes in saliva flow and the secretion of salivary secretory immunoglobulin A (sIgA). As exercise can induce changes in sympathetic and parasympathetic activity, it is a stimulus that can alter salivary secretions. For example, acute exercise of moderate intensity can increase sIgA secretion in both lower (Sari-Sarraf et al. 2007) and upper body (Leicht et al. 2011) modalities. Exercise intensity is central in altering salivary parameters, whereby exercise of demanding nature, such as interval training (Mackinnon et al. 1993) or marathon racing (Nieman et al. 2002), can reduce saliva flow rate and SIgA secretion. 
Salivary parameters have practical value as they are potential predictors of illness: reductions in sIgA, playing a significant part in mucosal immunity, have been associated with increased respiratory tract illness risk (Neville et al. 2008) and high training loads have been associated with a reduced SIgA secretion (Fahlman and Engels 2005; Neville et al. 2008; Leicht et al. 2012). However, the practical use is impacted by the high inter-individual variation in $\operatorname{sIg} \mathrm{A}$ (Neville et al. 2008; Leicht et al. 2012), making it difficult to define universal threshold values that may be related to illness risk. Whilst dehydration (Walsh et al. 2004) or upcoming illness (Neville et al. 2010) have an impact on salivary protein concentration, to date, the source of this inter-individual SIgA variation in healthy, adequately hydrated individuals remains obscure.

Cortisol impacts directly on immune cells: For example, infusion and correlation experiments show that cortisol induces lymphopenia (Tonnesen et al. 1987) and that cortisol can reduce $\mathrm{B}$ cell activation and proliferation (Cupps et al. 1985). In the context of mucosal immunity, this is particularly relevant as $\operatorname{IgA}$ is secreted from differentiated B cells (Lamm 1998). Cortisol is also susceptible to acute exercise stress (Hayes et al. 2010): whereas low intensity exercise is generally associated with no change or even reductions in cortisol concentration (Jacks et al. 2002; Hill et al. 2008), exercise performed at intensities at or higher than $\sim 60 \% \dot{V} \mathrm{O}_{2 \max }$ can acutely increase systemic (Hill et al. 2008) and salivary (Jacks et al. 2002; Hayes et al. 2015) cortisol concentration.

Hence, it appears that some common traits exist between cortisol and salivary parameters: they are both susceptible to exercise stress, and they play a role in host defence. However, whilst it has been reported that taxing sporting events inducing high cortisol concentrations also lead to perturbations of mucosal immunity (Nieman 1997), it is not known whether variations in cortisol concentration between individuals may be related to variations in the salivary response. The extent by which cortisol may explain some of the reported large inter-individual variations in salivary parameters (Neville et al. 2008; Leicht et al. 2012) is not known either. Furthermore, the upper body exercise modality has not received much attention in the field of mucosal immunity; however, the mucosal immune response and knowledge about the underlying mechanisms is especially important in those restricted to this modality due to their high infection susceptibility (Cardenas et al. 2004).

Therefore, this study investigates exercise of varying modality and intensity to analyse plasma cortisol concentration and salivary parameters in the subsequent $4 \mathrm{~h}$ period. Correlations between cortisol and salivary parameters within time points are examined. We hypothesise cortisol to explain some of the inter-individual variation in salivary parameters.

\section{Materials and methods}

Data were obtained as part of a study published previously (Leicht et al. 2016). As a consequence, the exercise intervention, participant, and exercise characteristics are identical between those studies. The study was approved by Loughborough University's Ethics committee. Written informed consent was obtained from all individual participants included in the study.

\section{Participants and experimental design}

Twelve recreationally trained male individuals volunteered to participate (age: $25 \pm 4$ years, body mass: $76 \pm 9 \mathrm{~kg}$, self-reported sporting activity $4.0 \pm 1.2 \mathrm{~h} \cdot \mathrm{week}^{-1}$, $\dot{V} \mathrm{O}_{2 \text { peak arms }}: 32.1 \pm 6.0 \mathrm{~mL} \cdot \mathrm{kg}^{-1} \cdot \mathrm{min}^{-1}, \dot{V} \mathrm{O}_{2 \text { peak legs }}$ : $46.2 \pm 6.8 \mathrm{~mL} \cdot \mathrm{kg}^{-1} \cdot \mathrm{min}^{-1}$ ). The participants visited the laboratory on six occasions for two preliminary and four main trials. Initially, body mass and stature were determined using scales (model 770, seca, Birmingham, UK) and a Leicester portable stadiometer (seca, Birmingham, UK), respectively. In the two preliminary trials (visits 1 and 2), peak oxygen consumption $\left(\dot{V} \mathrm{O}_{2 \text { peak }}\right)$ was determined during arm exercise (determining $\dot{V} \mathrm{O}_{2 \text { peak arms }}$ ) using an arm crank ergometer (Angio, Lode, Groningen, Holland) and during cycling exercise (determining $\dot{V} \mathrm{O}_{2}$ peak legs) using a cycle ergometer (Excalibur, Lode, Groningen, Holland) in a randomised order. For this, participants performed a graded exercise test to volitional exhaustion, with an initial power output of $35 \mathrm{~W}$ (arms) and $70 \mathrm{~W}$ (legs), respectively; power output was then increased every $3 \mathrm{~min}$ by $15 \mathrm{~W}$ (arms) or $30 \mathrm{~W}$ (legs) until exhaustion. Arm exercise was performed in a sitting position, the centre of the crank at shoulder level with arms slightly flexed at maximum reach, cycling with legs slightly flexed at maximum reach. Settings were noted and replicated for all main trials.

Main trials (visits 3-5) were performed in a randomised order after a $24 \mathrm{~h}$ food standardisation period, where participants consumed the same freely chosen diet for all main trials. Caffeine and exercise were not allowed $24 \mathrm{~h}$ before the experiments. To account for diurnal variations, exercise tests were performed in the morning (start: 07:45-09:15) for all participants and at the same time of day for each individual participant. Trials were performed at $21.9 \pm 0.6{ }^{\circ} \mathrm{C}$ and at $33 \pm 8 \%$ relative humidity. Main trials consisted of 45 min of steady state exercise as follows: (1) arm exercise at $60 \% \dot{V} \mathrm{O}_{2 \text { peak arms }}$; (2) moderate cycling at $60 \% \dot{V} \mathrm{O}_{2 \text { peak legs }}$; and (3) easy cycling at $60 \% \dot{V} \mathrm{O}_{2}$ peak arms. A 5 min warm-up was performed at $50 \%$ of the start load before each condition. Oxygen uptake $\left(\dot{V} \mathrm{O}_{2}\right)$ 
was determined at 5 min intervals and power output was adjusted if necessary. For all experiments, oxygen uptake was determined over 1 min periods using Douglas bags and a gas analyser (Servomex 1440, Servomex Ltd, Crowborough, UK), and heart rate was continuously monitored using a Polar RS400 (POLAR, Kempele, Finland) monitor. Participants further indicated their rating of perceived exertion (RPE) on a scale ranging from 6 to 20 (Borg 1982). Apart from the times when gas exchange was measured water during exercise was given ad libitum. Water intake in the $4 \mathrm{~h}$ post-exercise passive recovery period was recorded and replicated for the remaining main trials; food and drinks other than water were not allowed during the main trials. In the recovery period, participants were allowed to perform non-strenuous activities such as reading or writing, they were generally seated but were allowed to get up to for toilet visits. A further main trial (visit 6) was performed at the same time of day $(N=10)$, which consisted of a 45-min resting period instead of the exercise intervention for the collection of saliva samples at rest.

\section{Salivary and plasma analyses}

Saliva and plasma samples were obtained pre, post, $2 \mathrm{~h}$ post and $4 \mathrm{~h}$ post-exercise for all main trials. The pre-samples were collected $>60$ min after participants had woken up. Apart from the collection immediately after exercise, participants rested on a bed for $10 \mathrm{~min}$ before samples were taken. Saliva samples were taken first, immediately followed by the venepuncture.

Salivary analyses were performed as explained in detail previously (Leicht et al. 2011). In brief, participants rinsed their mouth with water 10 min before each saliva collection, they then provided an unstimulated timed saliva sample with their head slightly tilted forward and minimal orofacial movement, and saliva was immediately centrifuged ( $2 \mathrm{~min}$ at $13,400 \mathrm{rpm}$ ). The supernatant was stored at $-20^{\circ} \mathrm{C}$ for a maximum of 9 months. Saliva volume was estimated assuming saliva density to be $1.00 \mathrm{~g} \cdot \mathrm{mL}^{-1}$ (Cole and Eastoe 1988), and saliva flow rate was calculated from saliva volume and collection time. The sIgA concentration was determined in duplicate by sandwich enzyme-linked immunosorbent assay (ELISA), the within assay coefficient of variation being $2.8 \pm 3.5 \%$.

For venous blood sample collection, participants were lying in a supine position. Blood was collected into $\mathrm{K}_{3}$ EDTA monovette containers (Sarstedt, Nuembrecht, Germany) from a superficial vein of the forearm, drawn by separate venepunctures for each sample. Haematocrit was immediately determined for each sample using an automated haematology analyser (Coulter Ac.T 5diff OV; Beckman Coulter, High Wycombe, UK). Following centrifugation (10 min at $3000 \mathrm{rpm}$ and $4{ }^{\circ} \mathrm{C}$ ), plasma was stored at $-20{ }^{\circ} \mathrm{C}$ until analysis. Cortisol concentration was determined in duplicate by ELISA according to the manufacturer's instructions (DRG Instruments $\mathrm{GmbH}$, Marburg, Germany), with a within assay coefficient of variation of $2.7 \pm 2.7 \%$.

\section{Statistical analyses}

The SPSS 23.0 statistical package (SPSS Inc., Chicago IL, USA) was used for all statistical analyses. Means and standard deviations were computed for all variables, and normality was checked with the Shapiro Wilk test. Non-normal data were converted using square root (saliva flow rate) or logarithmic (cortisol, sIgA concentration and sIgA secretion rate) transformations which achieved normality for all variables. A repeated measures two-way (trial, time) analysis of variance (ANOVA) was conducted on cortisol, saliva flow rate, SIgA concentration and $\operatorname{sIgA}$ secretion rate. HuynhFeldt corrections were applied when sphericity was violated and Sidak adjustments applied for post hoc comparisons. Pearson's correlation coefficients $(R)$ and coefficients of determination $\left(R^{2}\right)$ were determined for bivariate relationships (cortisol vs saliva flow rate; cortisol vs SIgA concentration), and uncorrected $P$ values for these relationships are reported. Physiological exercise descriptors were analysed using a one-way (trial) repeated measures ANOVA or the non-parametric equivalents for non-normal and RPE data. Statistical significance was accepted at $P<0.05$.

\section{Results}

\section{Salivary parameters and plasma cortisol: relationships}

Negative relationships between plasma cortisol concentration and saliva flow rate were found for all trials at all time points (Fig. 1). Most common variation between the two variables across time points was observed during the easy cycle trial (Fig. 1I-K, $P<0.05$ ), whereas the common variation post-exercise and $2 \mathrm{~h}$ post-exercise was markedly reduced for arm exercise (Fig. 1B, C) and the moderate cycle trial (Fig. 1F, G).

Correlation coefficients between plasma cortisol concentration and SIgA concentration were predominantly positive but were considerably lower $\left(R^{2}\right.$ up to 0.24 ; Table 1$)$ than those found for the cortisol - saliva flow rate relationships ( $R^{2}$ up to 0.46 ; Fig. 1); none of these correlations reached significance $(P>0.05)$.

\section{Salivary parameters and plasma cortisol: time and trial effects}

SIgA concentration was decreased in the recovery period (main effect of time: $P<0.001$, Fig. $2 \mathrm{~A}$ ), whilst saliva 
Fig. 1 Correlations analysis of plasma cortisol concentration vs saliva flow rate for arm exercise, moderate (Mod.) and easy cycling pre, post, $2 \mathrm{~h}$ post and $4 \mathrm{~h}$ post-exercise. Raw data and linear regression lines

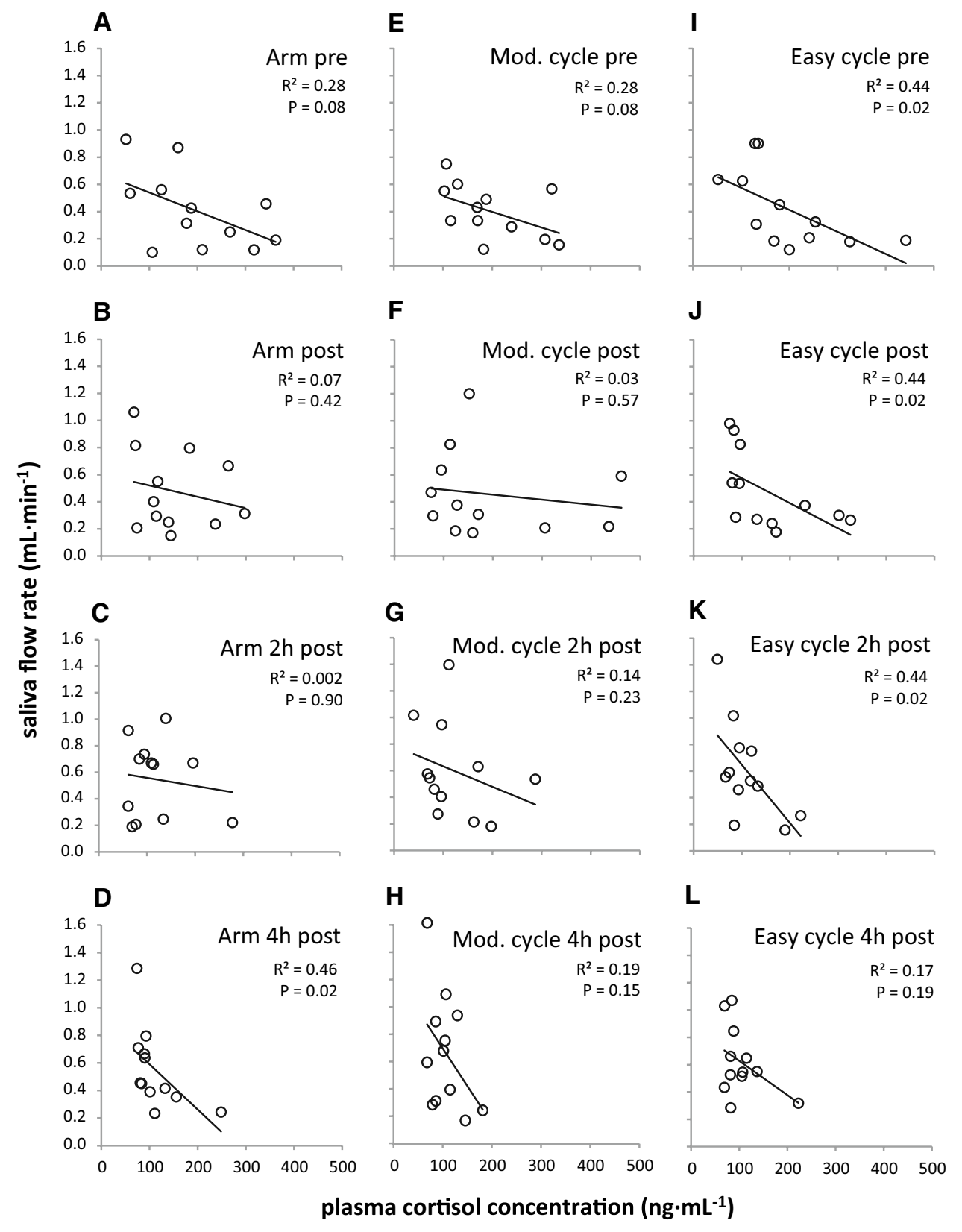

\begin{tabular}{llll}
\hline Time point & \multicolumn{2}{l}{ Trial } & \\
\cline { 2 - 4 } & Arm exercise & Moderate cycle & Easy cycle \\
\hline Pre & $R=0.34, R^{2}=0.12$ & $R=-0.04, R^{2}=0.002$ & $R=0.07, R^{2}=0.004$ \\
& $P=0.28$ & $P=0.90$ & $P=0.84$ \\
Post & $R=0.44, R^{2}=0.19$ & $R=0.24, R^{2}=0.06$ & $R=0.20, R^{2}=0.04$ \\
& $P=0.15$ & $P=0.46$ & $P=0.53$ \\
2 h Post & $R=0.24, R^{2}=0.06$ & $R=0.11, R^{2}=0.01$ & $R=0.15, R^{2}=0.02$ \\
& $P=0.46$ & $P=0.73$ & $P=0.64$ \\
& $R=0.49, R^{2}=0.24$ & $R=0.25, R^{2}=0.06$ & $R=0.32, R^{2}=0.10$ \\
& $P=0.11$ & $P=0.44$ & $P=0.31$ \\
\hline
\end{tabular}

Table 1 Bivariate correlations of plasma cortisol concentration vs SIgA concentration 
Fig. 2 Salivary responses $($ mean $\pm S D)$. Significant difference between ${ }^{\mathrm{a}}$ pre and ${ }^{\mathrm{b}}$ post $(P<0.05)$

flow rate increased as time progressed (main effect of time: $P<0.001$, Fig. $2 \mathrm{~B}$ ) with higher values in the recovery period. For all trials, sIgA secretion rate did not change over the course of the study (main effect of time: $P=0.16$; Fig. 2C). No effect of trial was found in any of the salivary parameters $(P=0.47-0.83)$, indicating that exercise did not alter these parameters when compared to the resting trial. The plasma cortisol concentration did not differ between trials $(P=0.30)$ and decreased over time, with a significant reduction at 2 and $4 \mathrm{~h}$ post $(P<0.001 ;$ Fig. 3$)$.

\section{Exercise response}

The exercise trials resulted in distinctively different physiological and psychophysiological responses, the lowest power output, heart rate and RPE values found for easy cycling. Arm exercise and easy cycling did not differ with regards to absolute oxygen uptake; arm exercise and moderate cycling did not differ with regards to relative oxygen uptake $(P>0.05$; Table 2$)$. Haematocrit did not differ significantly between trials $(P=0.23$; Table 3$)$.

\section{Discussion}

\section{Main findings}

This is the first study to analyse salivary parameters and plasma cortisol concentration and their relationship for different exercise intensities/modalities for an extended postexercise recovery period of $4 \mathrm{~h}$. The main finding was that plasma cortisol concentration explained some of the variance found in saliva flow (Fig. 1). This adds to the body of knowledge that has so far mainly focussed on neural regulation of salivary secretions (Chicharro et al. 1998; Proctor and Carpenter 2007). Second, increases in saliva flow rate were found over time, but sIgA secretion rate remained unchanged as this was accompanied by decreases in $\operatorname{SIgA}$ concentration (Fig. 2). These responses were also found in the resting trial and were not affected by exercise, a confirmation of earlier work indicating the presence of circadian rhythms for salivary markers (Dawes 1972, 1975). Finally, sIgA concentration decreases were accompanied by cortisol concentration decreases over time, and the relationships between those markers were mainly positive (Table 1). However, none of these relationships reached significance, implying that cortisol is not an appropriate surrogate marker for sIgA concentration and vice versa.
- Arm exercise $\square$ Moderate cycle $\square$ Easy cycle Rest
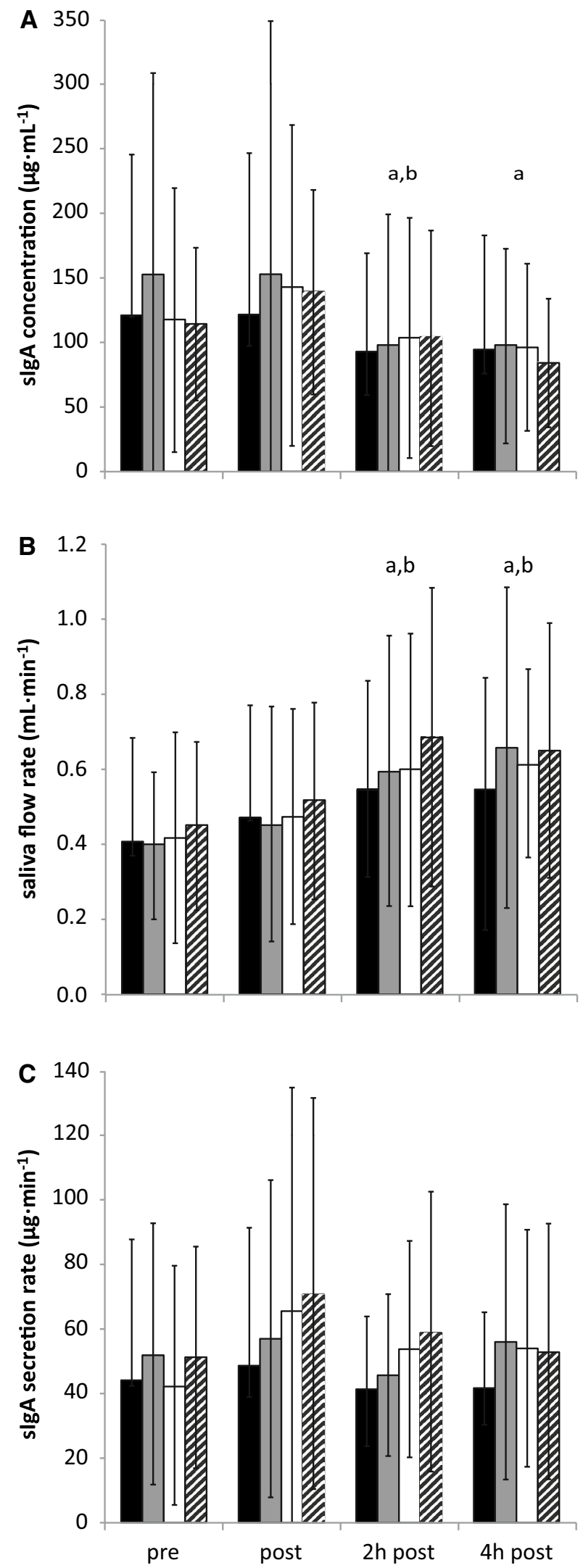


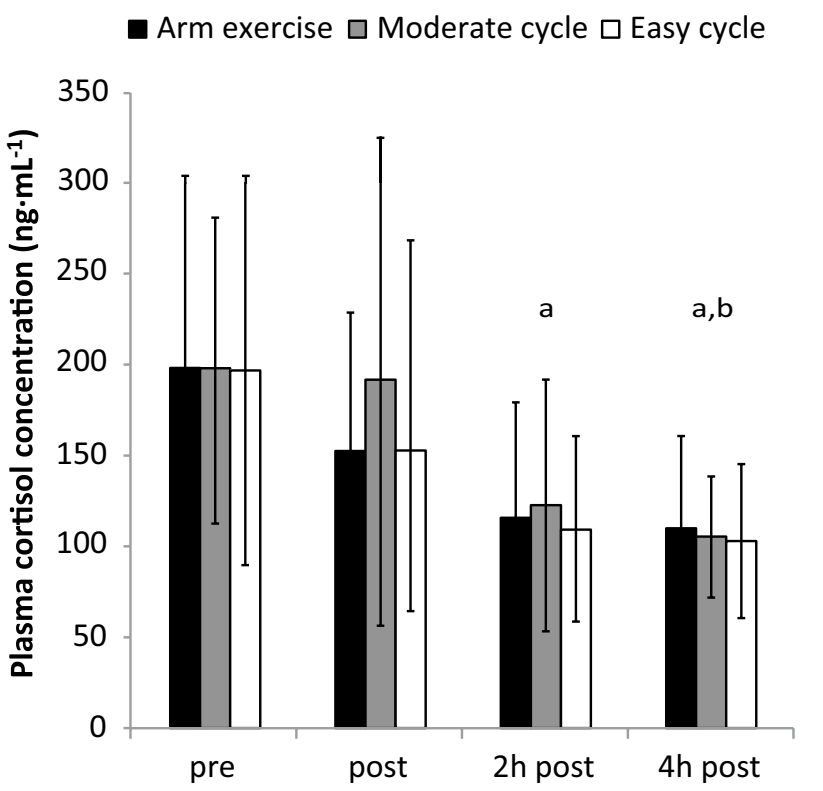

Fig. 3 Plasma cortisol responses (mean \pm SD). Significant difference between ${ }^{\mathrm{a}}$ pre and ${ }^{\mathrm{b}}$ post $(P<0.05)$

\section{Cortisol and its relationship with saliva flow}

The present findings show that some of the inter-individual variation of saliva flow rate can be explained by plasma cortisol concentration. This adds to earlier research comparing plasma cortisol and salivary parameters, showing the parallel fall in sIgA concentration and cortisol in the hours after awakening (Hucklebridge et al. 1998). Importantly, the present findings do not only support the established facts that circadian variations exist for cortisol (Dimitrov et al. 2009), saliva flow rate (Dawes 1975) and $\operatorname{sIgA}$ concentration ( $\mathrm{Li}$ and Gleeson 2004), with the fall of cortisol coinciding with the rise in saliva flow rate and the fall in SIgA concentration during the course of the day. It is crucial to note that a relationship between cortisol and saliva flow rate can be
Table 3 Haematocrit (\%) in response to the four main trials

\begin{tabular}{lllll}
\hline Time point & Trial & & & \\
\cline { 2 - 5 } & Arm exercise & Moderate cycle & Easy cycle & Rest \\
\hline Pre & $43.0 \pm 2.7$ & $43.3 \pm 2.7$ & $43.1 \pm 3.0$ & $41.1 \pm 2.9$ \\
Post & $45.0 \pm 2.9^{*}$ & $44.9 \pm 3.0^{*}$ & $44.3 \pm 2.7^{*}$ & $43.1 \pm 2.3^{*}$ \\
2 h Post & $43.3 \pm 2.9$ & $43.7 \pm 3.1$ & $43.5 \pm 3.3$ & $41.9 \pm 2.3$ \\
4 h Post & $42.9 \pm 3.1$ & $43.0 \pm 2.4$ & $43.1 \pm 2.7$ & $42.1 \pm 2.5$ \\
\hline
\end{tabular}

*Significant difference to other time points $(P<0.05)$

observed between participants for individual time points as well (hence excluding the factor circadian variation). This means that individuals with high cortisol concentrations tend to exhibit lower saliva flow rates. It is furthermore interesting that the negative relationships between cortisol concentration and saliva flow rate are less pronounced immediately post and $2 \mathrm{~h}$ post the two trials performed at moderate exercise intensity (arm exercise: Fig. 1B, C and moderate cycling: Fig. 1F, G). This is in contrast to easy cycling, where clear negative relationships are found throughout the analysed time points (Fig. 1I-L). Even though not measured in the current study, we speculate that the exercise intensity of these two trials was high enough to stimulate factors known to modulate saliva flow, such as adrenergic or cholinergic pathways (Proctor et al. 2003) or increased autonomic nerve activity (Carpenter et al. 2000), hence overriding the modulating effect of cortisol alone. The present correlations analysis also shows that easy cycling serves as an appropriate control trial with respect to the cortisol/saliva flow relationships, as they appear to remain unaffected by this exercise intervention.

The implication of cortisol in the regulation of saliva flow seems intriguing and the presented data provide a good starting point to discuss this suggestion. As many salivary parameters are expressed as secretion rates which take saliva flow rate into account, further investigations into the potential modulating role of cortisol have direct relevance
Table 2 Physiological and psychophysiological exercise descriptors

\begin{tabular}{llll}
\hline Parameter & \multicolumn{2}{l}{ Trial } & \\
\cline { 2 - 4 } & Arm exercise & Moderate cycle & Easy cycle \\
\hline Power output $(\mathrm{W})$ & $73 \pm 14^{*}$ & $148 \pm 30^{*}$ & $101 \pm 25^{*}$ \\
Heart rate $\left(b \cdot \mathrm{min}^{-1}\right)$ & $141 \pm 9^{*}$ & $150 \pm 12^{*}$ & $123 \pm 11^{*}$ \\
Average RPE & $13.3(12.6,13.9)$ & $13.2(12.7,14.0)$ & $10.6(10.0,11.0)^{*}$ \\
$\dot{\mathrm{V}} \mathrm{O}_{2}\left(\mathrm{~L} \cdot \mathrm{min}^{-1}\right)$ & $1.50 \pm 0.28$ & $2.16 \pm 0.34^{*}$ & $1.50 \pm 0.28$ \\
$\% \dot{\mathrm{V}} \mathrm{O}_{2 \text { peak arms }}$ & $62.3 \pm 1.4$ & - & $62.3 \pm 1.1$ \\
$\% \dot{\mathrm{V}} \mathrm{O}_{2 \text { peak cycling }}$ & - & $62.3 \pm 1.0$ & $43.2 \pm 5.4^{*}$ \\
\hline
\end{tabular}

Data indicate mean $\pm \mathrm{SD}$ or median (lower quartile, upper quartile)

$R P E$ rating of perceived exertion

*Significant difference to both other trials $(P<0.05)$ 
to the investigator of salivary secretions. It is worth pointing out that cortisol has not been considered to be potentially involved in the regulation of saliva flow previously; which has so far been attributed to autonomic neural regulation only (Chicharro et al. 1998; Proctor and Carpenter 2007). Nonetheless, the evidence presented here is correlational in nature, and causal evidence must now be obtained to substantiate this suggestion. This may involve cortisol infusion experiments to answer the cause and effect question with greater certainty and to establish the extent by which cortisol may modulate salivary secretions.

Approaching the problem from an alternative, exercisebased angle, future research may consider focussing on conducting exercise interventions in the afternoon. At this time, cortisol concentrations are relatively stable (Hucklebridge et al. 1998; Dimitrov et al. 2009) and do not fall following their circadian rhythm as they do in the morning, which may cancel out the effects of an intervention aimed at increasing cortisol concentrations. Exercise in the afternoon may hence induce a more pronounced rise in cortisol concentrations (Dimitriou et al. 2002), allowing the investigation of its effect on saliva flow and salivary secretions. It has also been suggested that the diurnal variation of cortisol and testosterone may impact on strength training adaptations: training performed in the afternoon is possibly more effective due to lower cortisol concentrations and more pronounced testosterone responses at this time of day (Hayes et al. 2010). Similarly, it could be speculated that performing exercise in the afternoon with its greater capacity to increase cortisol levels has a more pronounced impact on immunity than exercise in the morning. However, this must be substantiated with future experiments, as in the case of mucosal immunity, the limited existing evidence does not support this speculation (Dimitriou et al. 2002).

\section{Moderate intensity exercise and salivary secretory immunoglobulin A}

Previous data suggest that submaximal exercise can increase sIgA secretion rate (Sari-Sarraf et al. 2007; Leicht et al. 2011). A better protection against invading pathogens could hence be hypothesised based on the finding that reductions in sIgA increase the upper respiratory infection risk (Neville et al. 2008). However, these aforementioned studies (SariSarraf et al. 2007; Leicht et al. 2011) were limited to a postexercise monitoring period of up to $30 \mathrm{~min}$. This short postexercise observation period may have been a limitation that the current study aimed to overcome, and one of the aims of the present study was hence to monitor salivary parameters over a relatively long post-exercise period to establish how long any effects of acute submaximal exercise may last.

None of the exercise interventions in the current study significantly altered any of the salivary parameters in comparison to the responses observed in the resting trial. In light of this, it is important to point out that the literature is ambiguous with respect to the acute sIgA secretion response to exercise, with $\operatorname{sIg}$ A secretion rate reported to increase (Sari-Sarraf et al. 2007; Leicht et al. 2011) or to remain unaffected (Bishop et al. 2000; Reid et al. 2001; Sari-Sarraf et al. 2006; Davison 2011) by submaximal exercise interventions. Interestingly, sIgA concentrations decreased over time, with higher values in the morning, falling over the course of the day. These reductions in SIgA concentration over time can be related to the increase in saliva flow causing a diluting effect. Saliva flow rate hence affects $\operatorname{sg}$ A concentration without affecting $\operatorname{sIg}$ A secretion rate, which is in line with existing literature (Bishop et al. 2000; Reid et al. 2001). Whilst the present results do not confirm an increase in $\operatorname{sIgA}$ secretion rate for any exercise trial, they confirm previous work (Davison 2011) pointing out the importance to consider circadian variations in markers of mucosal immunity. Previous evidence investigating sIgA metabolism (MacKinnon and Jenkins 1993; Reid et al. 2001; Sari-Sarraf et al. 2006) without accounting for circadian variation may hence have to be revisited, especially when investigating exercise of a long duration (Bishop et al. 2000; Nieman et al. 2002) where such variation may have the largest impact.

A final aim of the current study was to investigate the effect of exercise modality. This is an important issue should previous findings around mucosal immunity that were predominantly derived from lower body exercise modalities (Bishop et al. 2000; Reid et al. 2001; Sari-Sarraf et al. 2006, 2007; Davison 2011) be applied to individuals restricted to upper body exercise modalities. It is worth noting that whilst some studies have investigated acute mucosal immune responses for upper body exercise modalities (Leicht et al. 2011; Allgrove et al. 2012), we are not aware of studies comparing upper and lower body exercise modalities. With the caveat that exercise did not significantly alter markers of mucosal immunity in the present study, it is worth pointing out that there was also no difference in any salivary markers between exercise modalities (Fig. 2). This provides a good base for future investigations comparing these modalities, which should now study interventions that do induce changes in mucosal immunity affecting host protection, for example, by altering sIgA metabolism. Even though the current protocol was designed to fulfil this purpose, we must again stress that SIgA secretion rate was unaffected by exercise. For future investigations, we suggest to increase the exercise duration and/or intensity to reach this aim.

\section{Limitations}

This study employed an a posteriori design for data analysis (i.e., using data from a previously published study). Further confirmation of the present findings should be 
obtained using a priori study designs. To account for circadian variation more rigorously, future studies may want to narrow the testing start time to a smaller window than the one presently chosen $(90 \mathrm{~min})$. Future studies are also encouraged to record and document data such as waking time, and relate data collection time points to waking time, especially relevant when tracking cortisol or sIgA responses immediately after awakening (Hucklebridge et al. 1998). We did not record these data in detail as all first samples were collected $>30$ min following awakening, which is the time frame after which the acute awakening response of $\operatorname{sIg} \mathrm{A}$ and cortisol appears to level off (Hucklebridge et al. 1998).

\section{Conclusion}

This study shows that plasma cortisol concentration explains some of the inter-individual variance found in saliva flow. Furthermore, the results demonstrate that circadian effects must be considered for salivary flow rate, which impacts on the concentration of any salivary compounds such as sIgA.

Acknowledgements To all participants we are thankful for their time and willingness to participate in this study. This research was supported by the Peter Harrison Centre for Disability Sport and the National Institute for Health Research (NIHR) Leicester Biomedical Research Centre. The views expressed are those of the authors and not necessarily those of the NHS, the NIHR or the Department of Health.

Author contributions All authors conceived and designed research, read and approved the manuscript. CL conducted experiments, analysed data, and wrote the manuscript.

\section{Compliance with ethical standards}

Conflict of interest The authors declare no conflict of interest.

Ethical approval All procedures performed in studies involving human participants were in accordance with the Ethical standards of the institutional committee and with the 1964 Helsinki declaration and its later amendments.

Open Access This article is distributed under the terms of the Creative Commons Attribution 4.0 International License (http://creativeco mmons.org/licenses/by/4.0/), which permits unrestricted use, distribution, and reproduction in any medium, provided you give appropriate credit to the original author(s) and the source, provide a link to the Creative Commons license, and indicate if changes were made.

\section{References}

Allgrove JE, Chapman M, Christides T, Smith PM (2012) Immunoendocrine responses of male spinal cord injured athletes to 1-hour self-paced exercise: pilot study. J Rehabil Res Dev 49:925-933
Bishop NC, Blannin AK, Armstrong E et al (2000) Carbohydrate and fluid intake affect the saliva flow rate and IgA response to cycling. Med Sci Sports Exerc 32:2046-2051

Borg GA (1982) Psychophysical bases of perceived exertion. Med Sci Sports Exerc 14:377-381

Cardenas DD, Hoffman JM, Kirshblum S, McKinley W (2004) Etiology and incidence of rehospitalization after traumatic spinal cord injury: a multicenter analysis. Arch Phys Med Rehabil 85:1757-1763

Carpenter GH, Proctor GB, Anderson LC et al (2000) Immunoglobulin A secretion into saliva during dual sympathetic and parasympathetic nerve stimulation of rat submandibular glands. Exp Physiol 85:281-286

Chicharro JL, Lucia A, Perez M et al (1998) Saliva composition and exercise. Sports Med 26:17-27

Cole AS, Eastoe JE (1988) Biochemistry and oral biology. Wright, London

Cupps TR, Gerrard TL, Falkoff RJ et al (1985) Effects of in vitro corticosteroids on B cell activation, proliferation, and differentiation. J Clin Invest 75:754-761. https://doi.org/10.1172/ JCI111757

Davison G (2011) Innate immune responses to a single session of sprint interval training. Appl Physiol Nutr Metab 36:395-404. https:// doi.org/10.1139/h11-033

Dawes C (1972) Circadian rhythms in human salivary flow rate and composition. J Physiol 220:529-545

Dawes C (1975) Circadian rhythms in the flow rate and composition of unstimulated and stimulated human submandibular saliva. J Physiol 244:535-548

Dimitriou L, Sharp NC, Doherty M (2002) Circadian effects on the acute responses of salivary cortisol and IgA in well trained swimmers. Br J Sports Med 36:260-264

Dimitrov S, Benedict C, Heutling D et al (2009) Cortisol and epinephrine control opposing circadian rhythms in T cell subsets. Blood 113:5134-5143. https://doi.org/10.1182/blood-2008-11-190769

Fahlman MM, Engels HJ (2005) Mucosal IgA and URTI in American college football players: a year longitudinal study. Med Sci Sports Exerc 37:374-380

Hayes LD, Bickerstaff GF, Baker JS (2010) Interactions of cortisol, testosterone, and resistance training: influence of circadian rhythms. Chronobiol Int 27:675-705. https://doi.org/10.3109/0742052100 3778773

Hayes LD, Grace FM, Baker JS, Sculthorpe N (2015) Exercise-induced responses in salivary testosterone, cortisol, and their ratios in men: a meta-analysis. Sports Med 45:713-726. https://doi.org/10.1007/ s40279-015-0306-y

Hill EE, Zack E, Battaglini C et al (2008) Exercise and circulating cortisol levels: the intensity threshold effect. J Endocrinol Invest 31:587-591. https://doi.org/10.1007/BF03345606

Hucklebridge F, Clow A, Evans P (1998) The relationship between salivary secretory immunoglobulin A and cortisol: neuroendocrine response to awakening and the diurnal cycle. Int J Psychophysiol 31:69-76

Jacks DE, Sowash J, Anning J et al (2002) Effect of exercise at three exercise intensities on salivary cortisol. J Strength Cond Res $16: 286-289$

Lamm ME (1998) Current concepts in mucosal immunity. IV. How epithelial transport of IgA antibodies relates to host defense. Am J Physiol 274:G614-7

Leicht CA, Bishop NC, Goosey-Tolfrey VL (2011) Mucosal immune responses to treadmill exercise in elite wheelchair athletes. Med Sci Sports Exerc 43:1414-1421. https://doi.org/10.1249/ MSS.0b013e31820ac959

Leicht CA, Bishop NC, Paulson TAW et al (2012) Salivary immunoglobulin A and upper respiratory symptoms during five months of 
training in elite tetraplegic athletes. Int J Sports Physiol Perform 7:210-217

Leicht CA, Paulson TAW, Goosey-Tolfrey VL, Bishop NC (2016) Arm and intensity-matched leg exercise induce similar inflammatory responses. Med Sci Sports Exerc 48:1161-1168. https:// doi.org/10.1249/MSS.0000000000000874

Li TL, Gleeson M (2004) The effect of single and repeated bouts of prolonged cycling and circadian variation on saliva flow rate, immunoglobulin A and alpha-amylase responses. J Sports Sci 22:1015-1024

MacKinnon LT, Jenkins DG (1993) Decreased salivary immunoglobulins after intense interval exercise before and after training. Med Sci Sports Exerc 25:678-683

Mackinnon LT, Ginn E, Seymour GJ (1993) Decreased salivary immunoglobulin A secretion rate after intense interval exercise in elite kayakers. Eur J Appl Physiol Occup Physiol 67:180-184

Neville V, Gleeson M, Folland JP (2008) Salivary IgA as a risk factor for upper respiratory infections in elite professional athletes. Med Sci Sports Exerc 40:1228-1236. https://doi.org/10.1249/ MSS.0b013e31816be9c3

Neville V, Pain MT, Kantor J, Folland JP (2010) Influence of crank length and crank-axle height on standing arm-crank (grinding) power. Med Sci Sports Exerc 42:381-387. https://doi.org/10.1249/ MSS.0b013e3181b46f3a

Nieman DC (1997) Risk of upper respiratory tract infection in athletes: an epidemiologic and immunologic perspective. J Athl Train $32: 344-349$
Nieman DC, Henson DA, Fagoaga OR et al (2002) Change in salivary $\operatorname{IgA}$ following a competitive marathon race. Int J Sports Med 23:69-75. https://doi.org/10.1055/s-2002-19375

Proctor GB, Carpenter GH (2007) Regulation of salivary gland function by autonomic nerves. Auton Neurosci 133:3-18. https://doi. org/10.1016/j.autneu.2006.10.006

Proctor GB, Garrett JR, Carpenter GH, Ebersole LE (2003) Salivary secretion of immunoglobulin A by submandibular glands in response to autonomimetic infusions in anaesthetised rats. $\mathrm{J}$ Neuroimmunol 136:17-24

Reid MR, Drummond PD, Mackinnon LT (2001) The effect of moderate aerobic exercise and relaxation on secretory immunoglobulin A. Int J Sports Med 22:132-137

Sari-Sarraf V, Reilly T, Doran DA (2006) Salivary IgA response to intermittent and continuous exercise. Int J Sports Med 27:849855. https://doi.org/10.1055/s-2006-923777

Sari-Sarraf V, Reilly T, Doran DA, Atkinson G (2007) The effects of single and repeated bouts of soccer-specific exercise on salivary IgA. Arch Oral Biol 52:526-532. https://doi.org/10.1016/j.archo ralbio.2006.11.016

Tonnesen E, Christensen NJ, Brinklov MM (1987) Natural killer cell activity during cortisol and adrenaline infusion in healthy volunteers. Eur J Clin Invest 17:497-503

Walsh NP, Laing SJ, Oliver SJ et al (2004) Saliva parameters as potential indices of hydration status during acute dehydration. Med Sci Sports Exerc 36:1535-1542 